

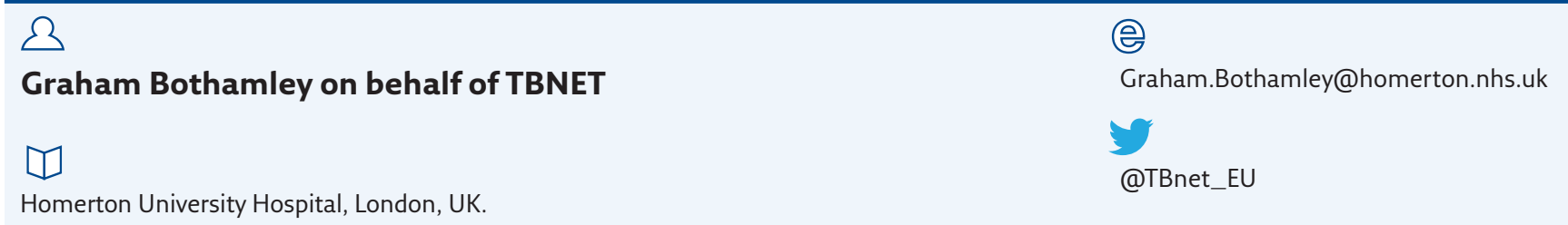

\section{The Tuberculosis Network European Trials Group (TBNET): new directions in the management of tuberculosis}

The Tuberculosis Network European Trials Group (TBNET) is the largest clinical research organisation in Europe. Educational activities include the TBNET Academy and the European Advanced Course in Clinical Tuberculosis. Four of their publications are reviewed to show how the clinical management of tuberculosis is changing.

@ ERSpublications

Learn about @TBnet_EU, the largest clinical research organisation in Europe http://ow.ly/NHTh30e53KE
Cite as: Bothamley G. The Tuberculosis Network European Trials Group (TBNET): new directions in the management of tuberculosis. Breathe 2017; 13: e65-e71.
25 years ago, World Tuberculosis Day was launched by the International Union Against Tuberculosis to commemorate the centenary of the discovery of the tubercle bacillus by Robert Koch. At that time, there were an estimated 10 million cases of tuberculosis (TB) worldwide with 1.8 million deaths. In the latest World Health Organization (WHO) report, there were an estimated 10.4 million TB cases with 1.4 million deaths. Even in the WHO European Region, the number of TB cases is the same as in 1987 [1]. The European Region has the fastest rate of increase in multidrug-resistant (MDR)-TB (i.e. strains resistant to both isoniazid and rifampicin) of all WHO regions. The probability that a pulmonologist will meet someone with TB is therefore much the same as for their predecessors, with the added problem that the choice of treatment may not be so easy.
The Tuberculosis Network European Trials Group (TBNET) (www.tb-net.org) was formed in 2006 to promote clinical research in TB, support TB education, and allow for the sharing and development of ideas and research protocols [2]. TBNET has two daughter organisations: ptbnet for addressing the same activities in children and NTM-NET for diseases caused by nontuberculous mycobacteria. From its inception with 41 physicians from 12 European countries, TBNET has grown to an organisation with almost 700 members from across the world, the majority practising in the European Union. With 54 peer-reviewed publications to date, TBNET has become the largest European research organisation in TB. In 2008, its chair and vice chair created the first Clinical Research Collaboration (CRC) with the European Respiratory Society. The latest CRC addresses the clinical aspects of MDR-TB. 
Education has always been a high priority for TBNET. The first TBNET Academy was held in 2011 in Austria, as a forum for young clinicians and researchers to exchange ideas and learn from each other under expert guidance and mentorship. Participants were grouped into five sections (clinical TB, immunology, microbiology, molecular biology and epidemiology with public health) to present jointly the state of the art in their field. Teaching is also given on writing research protocols and papers, along with how to give an effective presentation. TBNET Academies have been held in Moldova, Ukraine and Armenia, so that local participants may interact with others from across Europe. For more established clinicians, TBNET holds the European Advanced Course in Clinical Tuberculosis in collaboration with the International Union Against Tuberculosis and Lung Disease (Europe), Filha and the Karolinska Institute.

This review will look at a sample of four recent TBNET publications that are impacting the management of TB and will continue to do so over the next decade.

\section{Interferon $-\gamma$ release assays}

Contact tracing in TB is a necessary part of TB control. Firstly, such contacts may already have active TB (so-called prevalent cases at the point of screening) and most will have symptoms. However, previous studies have shown that $\sim 7 \%$ of those with active disease have no symptoms [3]. Screening tests need to have high sensitivity in order to ensure that no active case is missed [4]. The TBNET studyby ZellWeger et al. [5] indicated that at the first screening of contacts of pulmonary TB in Europe, active disease occurred in $0.5 \%$ (25 out of 5020), of whom $78 \%$ had symptoms. In this scenario, a positive interferon $\gamma$ release assay (IGRA) with or without a positive tuberculin skin test was used to indicate those who had no symptoms but merited chest radiography and further investigation. Even so, three of these "prevalent" cases had a negative IGRAs, which is consistent with the finding of an $80 \%$ sensitivity of IGRAs in active TB [6].

Secondly, although contacts may not have active TB, they may still be at risk of developing TB later. Latent tuberculosis infection (LTBI) (or longlasting TB immunity [7]) is defined by a positive tuberculin skin test or IGRA in the absence of active TB. Active TB is thought to develop in $10 \%$ with LTBI. This figure was based on studies in highincidence communities in the 1950s and 1960s, when re-infection would also have been high; those with radiographic abnormalities suggesting self-healed disease were included [8]. In current clinical practice, most contacts have a normal chest radiography. The TBNET study showed that nowadays, active TB develops in 16 (3.2\%) out of 494 with LTBI who did not receive preventive treatment but in just three $(0.6 \%)$ out of 481 who received preventive treatment and five $(0.16 \%)$ out of 3141 with a negative IGRA (consistent with background infection rates for communities with TB). A negative IGRA was of most value in stopping further investigations and reassuring contacts that they would not develop active TB from their family contact. The risk of TB was greater (3.3 per 100 person-years) in those with HIV co-infection and a positive IGRA, but only if the virus could be detected in their blood and they lived in high or medium TB incidence countries [9]. Preventive treatment is, therefore, effective in reducing the number of cases of active TB. However, not everyone so treated will benefit [10]. These data cannot be used to estimate the risk of developing active TB when screening other "high-risk" populations. Close or household contact with an infectious case of TB gives a much higher likelihood of developing active TB compared to those with no known contact [11].

From this study and similarly large European studies, which have shown much the same results, we can now estimate the potential yield of active TB from contact tracing $(0.5 \%)$ and the value of preventive treatment in a low incidence area (number needed to treat 30.4). From the median counts and estimating that half of all TB cases are pulmonary, you are likely to give preventive treatment to as many contacts as patients you treat for active TB. Immunological tests for latent infection are relevant in the early diagnosis of asymptomatic active TB, unless a chest radiograph and the measurement of inflammatory markers are usual for contacts (a tuberculin skin test might be easier in children than an IGRA). Follow-up of those with a positive IGRA after the initial clinic visit depends on the local resources available; an "inform and advise" leaflet of the symptoms of TB may be sufficient [12].

\section{Molecular drug-resistance testing}

MDR-TB is a significant problem, especially in Eastern European countries. Genetic tests for resistance are already widely used; microbiologists and practising TB physicians have assessed the clinical implications of these tests, as in the TBNET consensus statement by Domínguez et al. [13]. The use of DNA-based tests, such as Xpert MTB/ RIF (Cepheid, Sunnyvale, CA, USA), can identify the presence of Mycobacterium tuberculosis and whether there are any of the common mutations associated with rifampicin resistance in $r p o B$, the $\beta$-subunit of RNA polymerase (a constituent of the enzyme complex that transcribes RNA from DNA). Rifampicin monoresistance is a rare entity and most have concurrent isoniazid resistance, the definition of MDR-TB. The significance of combined isoniazid and rifampicin resistance is that patients with these strains require a more difficult and prolonged 
treatment, and have a significantly poorer outcome. Culture of M. tuberculosis is mostly available within Europe but subsequent drug-susceptibility testing (DST) may still take up to 2 months. Empirical regimens have been recommended in these circumstances. However, as noted in the discussion on short-course chemotherapy for MDR-TB, European strains are characterised by a much broader resistance pattern (table 1 and see later). Thus, the probability of using an ineffective regimen, which might also promote further drug resistance if the strain of $M$. tuberculosis is only sensitive to one the drugs used, is high. Individualised treatment of MDR-TB from the outset is therefore to be preferred and is of proven efficacy [14]; this requires rapid genetic tests.

The laboratory assessment of drug susceptibility is surprisingly difficult [15]. The critical concentration is the lowest amount of the drug that inhibits the growth of $>95 \%$ of $M$. tuberculosis not exposed to the drug and with no resistance. The minimum inhibitory concentration defines the lowest concentration of a drug that prevents measurable growth of an individual strain of M. tuberculosis and is usually assessed using drug concentrations derived from the critical concentration. It should be noted that, of all TB drugs, only isoniazid is bactericidal (acting on the cell wall like penicillin), and all other drugs are bacteriostatic; $M$. tuberculosis avoids death by entering a quiescent phase. "Sterilising" drugs, such as pyrazinamide, may not have a significant bacteriostatic effect but act to prevent entry into this quiescent phase, allowing other drugs to act effectively.

Drugs such as isoniazid and rifampicin show a clear distinction between susceptible and resistant strains [15]. Isoniazid resistance has two important levels, a low resistance due to inhA mutations that can be overcome with higher ( $900 \mathrm{mg}$ daily) doses, and a higher level of resistance due to mutations in
katG. These two mutations account for the majority of isoniazid resistance but there are many others that can lead to resistance [16].

The use of DNA probes for detecting resistance is easiest for rifampicin, where a single gene $(r p o B)$ with a limited number of potential mutations is the main target. Xpert MTB/RIF discordant results indicate that $6.2 \%$ have a negative test with rifampicin resistance, the majority being due to a mutation L511P (where lysine (L), the "normal" amino acid, at position 115 is replaced by proline $(P)$, the "new" amino-acid) in $r p o B$. However, there are still a few other mutations that can lead to resistance (e.g. those that cause greater expression and/or activity of efflux pumps which remove the drug from within the tubercle bacillus) [17]. Over the next few years, mapping of DST with genetic mutations identified by whole-genome sequencing (WGS) will begin to define how the phenotypic and genotypic resistance relate to each other. More importantly, the clear definition of a cure (see later) will determine the clinical value of WGS in shaping treatment regimens.

The important contribution of this consensus has been to highlight mutations that can be overcome by a selection of a different drug in the same class. Rifampicin resistance can be negated by the use of rifabutin (in the case of the D516mut mutation, where aspartate (D) at position 516 is replaced by any other amino acid (mut)). In terms of injectable drugs, rrs A1408G (where adenosine (A) would be the "normal" base at position 1408 of the DNA of the gene but it replace by guanine $(G)$ ) is associated with resistance to amikacin and kanamycin but low resistance to capreomycin, C1409T (where cytosine (C) at position 1409 is replaced by thymidine (T)) with low-level resistance to amikacin but high resistance to kanamycin and capreomycin, and eis mutations show low-level resistance to kanamycin and C14T mutations that may also show low-level resistance to amikacin.

Table 1 Reasons for drug resistance and how to avoid them

\begin{tabular}{|c|c|}
\hline Reasons for drug resistance & Action \\
\hline Adding a single drug to a failing regimen & Always add two or more drugs \\
\hline Inadequate or intermittent drug supply & $\begin{array}{l}\text { Political commitment } \\
\text { Maintain stocks }\end{array}$ \\
\hline Nonadherence & $\begin{array}{l}\text { Fixed drug combination tablets } \\
\text { Individual support (e.g. DOT) }\end{array}$ \\
\hline Quality of drugs for TB & Monitoring system \\
\hline Pharmacodynamics: how a drug affects the patient & Adverse effects should be managed promptly (e.g. antiemetics) \\
\hline $\begin{array}{l}\text { Pharmacokinetics: how human metabolism affects } \\
\text { the drug }\end{array}$ & $\begin{array}{l}\text { Check drug levels with high individual variation (e.g. rifampicin and } \\
\text { moxifloxacin } \# \text { ) }\end{array}$ \\
\hline
\end{tabular}

DOT: directly observed therapy. \#: although there is a 100-fold difference in plasma values of these drugs, routine measurement is not required for rifampicin except in isoniazid-resistant (pre-MDR-TB) disease and in those with fully sensitive strains who fail to show a significant improvement at 2 months (i.e. before starting the continuation phase of treatment). The usual effective dose of moxifloxacin for TB to achieve serum levels of 1-2 mg. $\mathrm{L}^{-1}$ is $600 \mathrm{mg}$, for which there is no formulation; $800 \mathrm{mg}$ is therefore required and only if there is a problem with this dose would levels need to be measured for a $400 \mathrm{mg}$ dose, to ensure efficacy. 
Table 2 Comparison of MDR-TB outcome measures (simplified)

\begin{tabular}{|c|c|}
\hline Outcome & LASERSON et al. [20] (2005) \\
\hline Died & $\begin{array}{l}\text { A patient who dies for any reason } \\
\text { during the course of treatment }\end{array}$ \\
\hline LTFU/default & $\begin{array}{l}\text { A patient whose treatment was } \\
\text { interrupted for } 2 \text { consecutive } \\
\text { months or more }\end{array}$ \\
\hline Treatment failed & $\begin{array}{l}\geq 2 \text { of } 5 \text { cultures in the final } \\
12 \text { months of treatment are } \\
\text { culture positive or if any of the } \\
\text { final } 3 \text { cultures are positive } \\
\text { OR } \\
\text { a clinical decision is made to } \\
\text { stop treatment due to a lack of } \\
\text { response }\end{array}$ \\
\hline
\end{tabular}

\section{Completed treatment}

Cured

Treatment success

\section{Undeclared}

Completed treatment but does not meet the definition of cure

Transfer out included as a separate element

Completed treatment according to protocol with $\geq 5$ negative cultures in the last 12 months or 1 positive culture with 3 subsequent cultures taken $\geq 30$ days apart

$$
\text { separate element }
$$

thent

WHO [23] (2014)

A patient who dies for any reason during the course of treatment

A patient whose treatment was interrupted for 2 consecutive months or more

Treatment terminated or need for permanent regimen change of at least two anti-TB drugs because of lack of conversion by the end of the intensive phase

bacteriological reversion in the continuation phase after conversion to negative evidence of additional acquired resistance to fluoroquinolones or second-line injectable drugs adverse drug reactions

Treatment completed as recommended by the national policy without evidence of failure BUT no record that $\geq 3$ consecutive cultures taken $\geq 30$ days apart are negative after the intensive phase

Treatment completed as recommended by the national policy without evidence of failure AND record that $\geq 3$ consecutive cultures taken $\geq 30$ days apart are negative after the intensive phase

The sum of cured and treatment completed

A patient for whom no treatment outcome is assigned (this includes "transferred out" where outcome unknown)

$\leq 12$ months (included in annual report) 36 months outcome after treatment started
TBNET [24] (2016)

A patient who dies during the period of observation

Non-receipt of care 6 months after treatment initiation

A positive culture 6 months after the start of treatment or a relapse within 1 year after treatment completion

Unacceptable: a measure of process and not treatment outcome

A negative culture status at 6 months after the start of treatment and no positive culture thereafter AND no relapses within 1 year after treatment completion

Not applicable

Outcome not assessed due to transferred out and no outcome known no culture at 6 months no post-treatment assessment

6 months into treatment and 12 months after treatment completion (most often 32 months after treatment started)

LTFU: lost to follow-up.

\section{Short-course chemotherapy for MDR-TB}

A short-course, 9-month "Bangladesh" regimen for the treatment of MDR-TB was first mooted in 2010 [18]. The regimen consisted of gatifloxacin, clofazimine, ethambutol and pyrazinamide for the entire course, supplemented with kanamycin, prothionamide and high-dose isoniazid (900 mg daily) until the sputum smear converted from positive to negative. This study, together with other follow-up studies in different locations, indicated that half needed 4 months and the other $50 \%$ needed up to 7 months of the more intensive phase. The continuation phase was then for a further 5 months. In a meta-analysis, cure rates were substantially better than with the then standard 20-month treatment (90\% compared to $78 \%$, WHO criteria; see later) but relapses were common if there was resistance to fluoroquinolones or kanamycin [19]. Using the criteria of LASERSON 
et al. [20], which include relapse within $\geq 2$ years, the cure rate was closer to $79 \%$ [19].

The WHO recommendations for use of this shorter regimen required [19]:

- no previous treatment with any second-line drug for $>1$ month;

- DST/genetic testing to confirm sensitivity to fluoroquinolones AND injectable drugs;

OR

- resistance considered unlikely from

1) the DST of any index case;

2) representative surveillance data.

The use of high-dose isoniazid is expected to overcome isoniazid resistance due to inhA mutations and of prothionamide to overcome kat $G$ mutations. Fluoroquinolone resistance is tested using ofloxacin for DST but gyrA and gyrB (the gene products of which unwind DNA for replication) mutations are reasonable indications of resistance [13].

In the TBNET study by LANGE et al. [21], the first item of note is how rarely DST is currently performed for second-line drugs (an average of $54 \%$ in 1140 patients examined). Resistance to injectable drugs (37\%) and fluoroquinolones (33\%) was relatively common, while resistance to prothionamide, ethambutol and pyrazinamide was even higher at $\geq 60 \%$. From these data, only 48 (8\%) out of 1140 would have been eligible for short-course treatment in hospitals representative of the European experience of MDR-TB. Furthermore, DST was usually derived from phenotypic testing and therefore would not have been available at the time of diagnosis. A recent survey indicated that genetic testing for second-line drugs is available in $44-55 \%$ of TB institutes with a particular interest in TB at a median of 5-7 days [22] and that even first-line DST is frequently delayed beyond 2 months [23].

\section{Treatment outcome in MDR-TB}

Treatment outcome definitions in MDR-TB were first suggested by LASERSON et al. [20] and were distinct from the then current $\mathrm{WHO}$ guidelines for fully sensitive TB. The difference was recommended on several grounds: the importance of DST; the longer duration of treatment; and that two negative sputum samples at the end of treatment were inadequate as an indicator of cure. WHO then updated outcome definitions (table 2) with particular reference to MDR-TB [24]. However, when using the latter definition for a cohort of patients with MDR-TB, TBNET contributors GÜNTHER et al. [25] noted that extensively drugresistant (XDR)-TB appeared to have a better outcome than MDR-TB. This seemed intuitively incorrect and, therefore, an attempt was made to arrive at outcome definitions that reflected clinical realities (table 2) [26].

The TBNET analysis showed that WHO-defined treatment success was determined largely by treatment completion (a process outcome). In the proposed definition, relapse-free survival was a more significant measure. In the TBNET cohort, $44(14 \%)$ out of 318 with a negative culture at 6 months became culture-positive at a later stage. Furthermore, cure without relapse followed a more expected pattern of $61 \%$ in MDR-TB, $52 \%$ in those with pre-XDR-TB and 39\% in XDR-TB, compared to treatment success of $68 \%, 62 \%$ and $79 \%$, and cure of $31 \%, 27 \%$ and $24 \%$, respectively, using WHO definitions. Treatment failure was not identified in $50(57 \%)$ out of 88 by the WHO definition. One of the key problems in monitoring the treatment of MDR-TB is the failure to obtain sputum samples for assessing cure.

\section{Conclusion}

The clinical management of TB is evolving. LTBI requires greater understanding in order to predict who will develop active disease and new RNA signatures need clinical evaluation in large cohorts. WGS cannot yet define antibiotic resistance, apart from rifampicin and to a lesser extent isoniazid, but may provide an understanding for new therapeutic approaches (e.g. $\beta$-lactam use, inhibition of efflux pump genes and preventing dormancy to reduce the duration of TB treatment regimens). Randomised controlled trials to replace collation of cohort studies, even those with sufficient follow-up for effective outcome measures, will become even more important. TBNET remains committed to evaluating expert views and scientific advances, particularly as to how they affect and might improve the clinical management of TB.

\section{Conflict of interest}

None declared.

\section{References}

1. World Health Organization. Global Tuberculosis Control. WHO Report 2001. WHO/CDS/TB/2001.287. Geneva, WHO, 2001.
2. GiehI C, Lange C, Duarte R, et al. TBNET - collaborative research on tuberculosis in Europe. EurJ Micobiol Immunol 2012; 2: 264-274. 
3. Meijer J, Barnett GD, Kubik A, et al. Identification of sources of infection. Bull Int Union Tuberc 1971; 45: 5-54.

4. World Health Organization. High-priority target product profiles for new tuberculosis diagnostics: report of a consensus meeting. Geneva, Global TB Programme, 2014.

5. Zellweger JP, Sotgiu G, Block M, et al. Risk assessment of tuberculosis in contacts by interferon- $\gamma$ release assays (IGRAs): a TBNET study. Am J Respir Crit Care Med 2015; 191 1176-1184.

6. Pai M, Zwerling A, Menzies D. Systematic review: T-cell-based assays for the diagnosis of latent tuberculosis infection: an update. Ann Intern Med 2008; 149: 177-184.

7. Mack U, Migliori GB, Sester M, et al. LTBI: latent tuberculosis infection or lasting tuberculosis immune responses? Eur Respir J 2009; 33: 956-973.

8. Smieja M, Marchetti C, Cook D, et al. Isoniazid for preventing tuberculosis in non-HIV infected persons. Cochrane Database Syst Rev 1999; CD001363.

9. Sester M, van Leth F, Bruchfeld J, et al. Risk assessment of tuberculosis in immunocompromised patients. Am J Respir Crit Care Med 2014; 190: 1168-1176.

10. Sester $M$, van Crevel $R$, van Leth $F$, et al. Numbers needed to treat to prevent tuberculosis. Eur Respir J 2015 46: 1836-1838.

11. Horsburgh CR. Priorities for the treatment of latent tuberculosis infection in the United States. N Engl/ Med 2004 350: 2060-2067.

12. National Institute for Health and Care Excellence. Tuberculosis. NICE guideline [NG33] nice.org.uk/guidance/ ng33 Date last updated: May 2016

13. Domínguez J, Boettger EC, Cirillo D, et al. Clinica implications of molecular drug resistance testing for Mycobacterium tuberculosis: a TBNET/RESIST-TB consensus statement. Int J Tuberc Lung Dis 2016; 20: 24-42.

14. Olaru ID, Lange C, Indra A, et al. High rates of treatment success in pulmonary multidrug-resistant tuberculosis in individually tailored treatment regimens. Ann Am Thorac Soc 2016; 13: 1271-1278
15. Kim SJ. Drug-susceptibility testing in tuberculosis: methods and reliability of results. Eur Respir J 2005; 25: 564-569.

16. Vilcheze C, Jacobs WR. Resistance to isoniazid and ethionamide in Mycobacterium tuberculosis: genes mutations and causalities. Microbiol Spectrum 2013; 2: MGM2-0014-2013.

17. Kanji A, Hasan R, Zaver A, et al. Alternate efflux pump mechanism may contribute to drug resistance in extensively drug-resistant isolates of Mycobacterium tuberculosis. Int Mycobacteriol 2016; 5: Suppl. 1, S97-S98.

18. Van Deun A, Maug AKJ, Salim AH, et al. Short, highly effective, and inexpensive standardized treatment pf multidrug-resistant tuberculosis. Am J Respir Crit Care Med 2010; 182: 684-692.

19. World Health Organization. WHO Treatment guidelines for drugresistant tuberculosis - 2016 update. Geneva, WHO, 2016.

20. Laserson KF, Thorpe LE, Lemaine $V$, et al. Speaking the same language: treatment outcome definitions for multidrugresistant tuberculosis. Int J Tuberc Lung Dis 2005; 9: 640-645.

21. Lange C, Duarte $R$, Fréchet-Jachym $M$, et al. Limited benefit of the new shorter multidrug-resistant tuberculosis regimen in Europe. Am J Respir Crit Care Med 2016; 194: 1029-1031.

22. Bothamley G, Lange C. Availability of PCR and second line drugs in Europe. Eur RespirJ 2016; 48: Suppl. 60, OA1510.

23. Bothamley G, Lange C. Preventing MDRTB in Europe: drug sensitivities received after the initial phase of treatment. Eur Respir J 2016; 48: Suppl. 60, OA3512.

24. World Health Organization. Definitions and reporting framework for tuberculosis - 2013 revision (updated December 2014). WHO/HTM/TB/2013.2:7. Geneva, WHO, 2013.

25. Günther G, Lange C, Alexandru S, et al. Treatment outcomes in multidrug-resistant tuberculosis. N Engl J Med 2016; 375: 1103-1105.

26. Lange C, Abubakar I, Alffenaar JC, et al. Management of patients with multidrug-resistant/extensively drug-resistant tuberculosis in Europe: a TBNET consensus statement. Eur Respir J 2014; 44: 23-63. 